\title{
Modelo hidrológico para estimativa do escoamento superficial em áreas agrícolas
}

\author{
Fernando F. Pruski ${ }^{1}$, Lineu N. Rodrigues ${ }^{2} \&$ Demetrius D. da Silva ${ }^{3}$ \\ 1 UFV. CEP 36571-000, Viçosa, MG. Fone: (31) 3899-1912, Fax: (31) 3899-2735. E-mail: ffpruski@mail.ufv.br (Foto) \\ 2 Postdoctoral Research Engineer, Department of Biological Systems Engineering, University of Nebraska, Lincoln, NE, \\ USA. E-mail: lineun@unlserve.unl.edu \\ 3 UFV, CEP 36571-000, Viçosa, MG. Fone: (31) 3899-1904, Fax: (31) 3899-2735. E-mail: david@mail.ufv.br
}

Protocolo $134-31 / 10 / 2000$

\begin{abstract}
Resumo: Desenvolveu-se um modelo hidrológico para estimar o escoamento superficial em áreas sob condições agrícolas. O modelo é baseado na análise dos diversos processos associados ao balanço hídrico e nele se usa a equação de intensidade, duração e freqüência da precipitação para estimar a intensidade de precipitação e a equação de Green-Ampt modificada por Mein-Larson, para estimar a velocidade de infiltração permitindo, também, a consideração da interceptação pela cobertura vegetal e do armazenamento de água sobre a superfície do solo por meio de diferentes metodologias. O escoamento superficial começa após a capacidade de armazenamento sobre a superfície do solo ter sido preenchida, e é calculado subtraindo-se a velocidade de infiltração da água no solo da intensidade de precipitação durante o intervalo de tempo em que a intensidade da chuva é maior que a velocidade de infiltração. Resultados de um experimento de validação do modelo e duas aplicações práticas são apresentados, sendo que os resultados obtidos com o modelo foram muito próximos daqueles observados no campo.
\end{abstract}

Palavras-chave: balanço da água no solo, escoamento superficial, modelo hidrológico

\section{A hydrologic model to estimate the surface runoff in agricultural lands}

\begin{abstract}
A hydrologic model was developed to estimate the surface runoff under agricultural conditions. The model is process-based and uses the intensity-duration-frequency equation to calculate the rainfall intensity and the Green-Ampt-Mein-Larson equation to estimate the infiltration rate. The runoff begins after the maximum surface storage on the soil surface has been reached. It is calculated by subtracting the infiltration rate from the rainfall intensity during the interval of time that the rainfall intensity is greater than the infiltration rate. The model also allows the calculation of the deep percolation and the actual soil-water content. Results from a validation experiment and two practical applications of the model are also presented. The results obtained with the model were approximately the same as those observed in the field.
\end{abstract}

Key words: soil water balance, surface runoff, hydrologic model

\section{INTRODUÇÃO}

A resposta hidrológica de uma bacia hidrográfica a diferentes sistemas de uso e manejo do solo é importante no processo de modelagem do escoamento superficial em pequenas bacias hidrográficas e no projeto de sistemas de irrigação por aspersão, estando a eficiência da modelagem do comportamento hidrológico desta bacia na dependência da eficiência com que cada componente associado a este é obtido. Enquanto a precipitação e o escoamento superficial são facilmente mensuráveis, a infiltração e o armazenamento superficial constituem-se em variáveis de difícil quantificação, em muitas situações (Mohamoud et al., 1990).
A erosão do solo e os conseqüentes impactos na produtividade agrícola, na qualidade da água e no assoreamento de cursos e reservatórios d'água, são preocupações evidenciadas em todo o mundo e, embora diversas técnicas sejam disponíveis para controlar a erosão, a compreensão do complexo processo físico envolvido na erosão é fundamental para se disponibilizar medidas de controle mais práticas e econômicas.

Uma vez que a erosão hídrica está diretamente relacionada à precipitação e ao escoamento superficial, sua modelagem deve ser associada aos procedimentos usados para descrever a produção do escoamento superficial e seu movimento, tanto na encosta como concentrado em canais. Alguns modelos, como o WEPP (Flanagan \& Nearing, 1995) e o CREAMS (Knisel, 1980), 
usam simulação contínua para modelar a produção de escoamento superficial, o que requer grande quantidade de informações relacionadas ao clima e ao uso do solo associados às condições em estudo. Além disso, eles são sensíveis à evapotranspiração e à dinâmica do solo e simulam uma grande quantidade de pequenos eventos que podem não produzir escoamento superficial e perdas de solo expressivas. Desta forma, parece mais apropriado direcionar-se a atenção ao desenvolvimento de um modelo baseado em eventos considerando-se, como indicado, que a erosão é dominada somente por poucos eventos que ocorrem ao longo do ano (Morgan et al., 1998)

Uma fração do volume total precipitado é interceptada pela vegetação e o restante atinge a superfície do solo. $\mathrm{O}$ escoamento superficial ocorre quando a intensidade da precipitação excede a velocidade de infiltração da água no solo. Após a velocidade de infiltração ser excedida, a água começa a preencher as depressões existentes na superfície do solo e, na seqüência, começa o escoamento superficial (Linsley et al., 1975; Mohamoud et al., 1990).

O processo de infiltração é muito complexo, mesmo quando é assumido que o solo é um meio homogêneo com conteúdo inicial de umidade uniforme. Para muitos eventos de precipitação existe um período inicial durante o qual toda a precipitação se infiltra no solo. Durante este período e como a água se infiltra, a capacidade de infiltração decresce, até tornar-se menor que a intensidade de precipitação; a partir deste momento, a água começa a acumular-se sobre a superfície do solo e o escoamento superficial pode ocorrer. Para representar adequadamente um evento em que haja a produção de escoamento superficial, o hidrologista deve ser hábil não somente a predizer este instante mas, também, o declínio subseqüente na capacidade de infiltração (Mein \& Larson, 1973).

A rugosidade da superfície é uma propriedade dinâmica do solo que influencia muitos processos que ocorrem na superfície, afetando a infiltração, o armazenamento superficial, o escoamento superficial, a refletância solar, a liberação e o transporte de partículas associadas à erosão hídrica, bem como o perfil do vento sobre a superfície do solo (Potter, 1990).

O escoamento superficial é o principal processo associado à erosão hídrica. Embora o impacto das gotas de chuva desempenhe importante papel na liberação das partículas de solo, é o escoamento da água que promove o transporte das partículas de solo liberadas para áreas em que ocorre o escoamento concentrado e, dependendo da carga de sedimentos e da capacidade de transporte do escoamento é que ocorrerá a liberação ou a deposição dos sedimentos. Muitas das práticas para o controle da erosão são, efetivamente, práticas para o controle da velocidade e do volume de escoamento superficial. Muitas aproximações têm sido feitas visando a consideração dos efeitos do escoamento superficial no processo erosivo, variando desde a desconsideração dos efeitos diretos do escoamento superficial até aquelas que consideram o perfil e a velocidade de escoamento da água (Stone et al., 1996).

Estimativas dos valores máximos de escoamento superficial são necessárias tanto em bacias hidrográficas com ocupação agrícola como urbana (Bonta \& Rao, 1992). Dados de volume máximo de escoamento superficial são necessários em estudos de manejo do solo e da água para determinar a eficiência dos métodos de preparo e manejo do solo e no planejamento de irrigação suplementar (Pathak et al., 1989). Eles também são usados no projeto de diques, canais e outras estruturas para o controle da erosão. Se o objetivo é reter ou armazenar todo o escoamento superficial, o conhecimento do volume total de escoamento superficial é suficiente, mas se o problema é transferir o excesso de água de um lugar para outro, é necessário se conhecer a vazão de escoamento superficial, especialmente a vazão correspondente a um período de retorno particular (Schwab et al., 1981).

Bonta \& Rao (1992) mostraram a dificuldade em se aplicar a tecnologia disponível para estimar as vazões máximas de escoamento superficial, devido à imprecisão dos métodos normalmente usados e da grande discrepância obtida por diferentes projetistas que usem o mesmo procedimento; conseqüentemente, é de grande importância obter-se um método confiável para estimar o escoamento superficial, razão pela qual Pruski et al. (1997b) desenvolveram um procedimento para se calcular o volume máximo de escoamento superficial em localidades onde a equação de intensidade, duração e freqüência da precipitação é conhecida.

O objetivo deste trabalho foi desenvolver um modelo, baseado em princípios físicos, para se estimar o escoamento superficial em áreas sob condições agrícolas.

\section{METODOLOGIA}

As pressuposições seguintes foram feitas no desenvolvimento do modelo: (a) a chuva somente atinge a superfície do solo após a interceptação pela cobertura vegetal ter sido completada; e (b) a capacidade de armazenamento superficial não varia com o tempo.

Os componentes associados ao balanço hídrico foram calculados usando-se o seguinte procedimento: com o início da precipitação começa, simultaneamente, a interceptação pela cobertura vegetal, que representa a fração da precipitação retida pela cobertura vegetal e que, portanto, não atingirá a superfície do solo. A máxima interceptação pela cobertura vegetal pode ser: (a) estimada de um banco de dados, a partir de informações contidas em Woolhiser et al. (1990) e Morgan et al. (1998); ou (b) calculada pelo projetista - o cálculo pode ser feito a partir de um valor conhecido ou estimado da área foliar. Quando o cálculo é feito a partir de um valor conhecido de área foliar, a máxima interceptação pela cobertura vegetal é estimada em função do índice de área foliar (IAF) pela equação proposta por Ehlers (1989):

$$
\mathrm{ICV}=0,932+0,499 \mathrm{IAF}+0,0057 \mathrm{IAF}^{2}
$$

sendo:

$$
\mathrm{IAF}=\frac{\mathrm{A}_{\mathrm{p}} \mathrm{S}_{\mathrm{d}}}{10000}
$$

em que ICV - interceptação máxima pela cobertura vegetal, mm; $\mathrm{IAF}$ - índice de área foliar da cultura, $\mathrm{cm}^{2}$ de folhas $\mathrm{cm}^{-2}$ de solo; $A_{p}$ - área foliar por planta no tempo $t, \mathrm{~cm}^{2}$ de folhas planta ${ }^{-1} \mathrm{e}$ $\mathrm{S}_{\mathrm{d}}$ - densidade de plantas, plantas $\mathrm{m}^{-2}$. 
A Eq. 1 não pode ser aplicada quando $\mathrm{IAF}=0$. Para esta condição, o valor de ICV é zero. A área foliar máxima por planta no tempo té estimada pela equação de Gompertz (Baker et al., 1975):

$$
A_{p}=A_{p(\max )} \exp \left(-b_{p} \exp \left(-k_{p} D D T\right)\right)
$$

em que $A_{p(\max )}$ - máxima área foliar por planta, $\mathrm{cm}^{2}$ de folhas planta $^{-1} ; b_{p}, k_{p}$ - constantes adimensionais que determinam a posição e a distribuição da curva ao longo do eixo do tempo, respectivamente; e DTT - tempo térmico acumulado, graus-d.

Toda a precipitação é interceptada pela cobertura vegetal até o momento em que o total precipitado se iguala à lâmina de interceptação potencial, sendo a duração da interceptação pela cobertura vegetal $\left(\mathrm{t}_{(\mathrm{ICV})}\right)$ calculada resolvendo-se a equação

$$
\int_{0}^{t_{(I C V)}} i_{i} d t=I C V
$$

em que $i_{i}$ - intensidade instantânea de precipitação, $\mathrm{mm} \mathrm{h}^{-1}$; e $t_{\text {(ICV) }}$ - tempo de duração da interceptação pela cobertura vegetal, $\mathrm{min}$.

A intensidade de precipitação instantânea é obtida com as equações

$$
\mathrm{i}_{\mathrm{i}}=\mathrm{i}_{\mathrm{m}}\left(1-\frac{\mathrm{ct}}{\mathrm{t}+\mathrm{b}}\right)
$$

sendo:

$$
\mathrm{i}_{\mathrm{m}}=\frac{\mathrm{K} \mathrm{T}^{\mathrm{a}}}{(\mathrm{t}+\mathrm{b})^{\mathrm{c}}}
$$

em que $\mathrm{i}_{\mathrm{m}}$ - intensidade máxima média de precipitação, $\mathrm{mm} \mathrm{h}^{-1}$; $\mathrm{T}$ - período de retorno, anos; $\mathrm{t}$ - duração da precipitação, min; e K, a, b, c - parâmetros da equação de chuvas intensas relacionados à localidade de interesse.

A Eq. 6 é conhecida como a equação de intensidade, duração e freqüência da precipitação. Considerando a dificuldade que representa a obtenção dos parâmetros desta equação, Pruski et al. (1997a) desenvolveram um procedimento para a regionalização dos parâmetros $(K, a, b, c)$ da equação de intensidade, duração e frequência da precipitação para amplas áreas de abrangência. Com o uso deste procedimento, diversos autores obtiveram esses parâmetros para vários estados brasileiros. Pruski et al. (1997a) os obtiveram para o Paraná, Silva et al.(1999b) para São Paulo, Pruski et al. (1999) para Minas Gerais e Silva et al. (1999a) para o Espírito Santo e Rio de Janeiro.

A partir do momento em que toda a lâmina potencialmente interceptável pela cobertura vegetal é preenchida, a lâmina precipitada passa a ser transformada em infiltração e, a partir deste momento, tem-se que a velocidade de infiltração se iguala à intensidade instantânea de precipitação. Esta condição é mantida até que $\mathrm{i}_{\mathrm{i}}$ supere a velocidade de infiltração da água no solo.

A infiltração acumulada, que ocorre do momento em que cessa a interceptação até o início do empoçamento de água sobre a superfície do solo, pode ser estimada pela equação:

$$
I=\int_{t_{\text {(ICV) }}}^{t_{i(A R M)}} i_{i} d t
$$

em que I - infiltração acumulada, mm; e $\mathrm{t}_{\mathrm{i}(\mathrm{ARM})}$ - tempo de início de ocorrência da fase de armazenamento superficial, ou seja, tempo de início do empoçamento de água sobre a superfície do solo, min.

Diversos autores discutem, em seus trabalhos, a respeito de equações baseadas em processos físicos para predizer a infiltração e o escoamento superficial em condições de precipitações com taxas de aplicação variáveis. Mein \& Larson (1973) obtiveram uma equação para calcular o volume de água antes da saturação da superfície sob condições de precipitação constante usando, para tanto, uma base conceitual similar àquela da equação de Green-Ampt. Chu, em 1978, mostrou que esta relação é também válida para taxas variáveis de aplicação se a intensidade de aplicação é uma função do tempo (Slack, 1980). Para taxas variáveis, a equação de Green-Ampt-MeinLarson (GAML) pode ser reescrita como:

$$
\mathrm{VI}=\mathrm{K}_{\mathrm{s}}\left(1+\frac{\left(\theta_{\mathrm{s}}-\theta_{\mathrm{i}}\right) \mathrm{S}_{\mathrm{w}}}{\mathrm{I}(\mathrm{t})}\right)
$$

em que VI - velocidade de infiltração da água no solo, $\mathrm{mm} \mathrm{h}^{-1}$; $\mathrm{K}_{\mathrm{s}}$ - condutividade hidráulica do solo saturado, $\mathrm{mm} \mathrm{h}^{-1}$; $\theta_{\mathrm{s}}$ - umidade de saturação do solo, $\mathrm{cm}^{3}$ de água $\mathrm{cm}^{-3}$ de solo; $\theta_{\mathrm{i}}$ - umidade inicial do solo, $\mathrm{cm}^{3}$ de água $\mathrm{cm}^{-3}$ de solo; $\mathrm{S}_{\mathrm{w}}$ - potencial matricial da água no solo na frente de umedecimento, mm.c.a.

No tempo $t_{i(A R M)}$ tem-se o início do empoçamento da água sobre a superfície do solo e a velocidade de infiltração é expressa pela equação de GAML; portanto, o armazenamento (empoçamento) de água sobre a superfície do terreno somente começa a partir do momento em que a intensidade instantânea de precipitação supera a velocidade de infiltração da água no solo.

A capacidade (lâmina máxima) de armazenamento superficial (ARM), em mm, pode ser calculada usando-se o método empírico proposto por Onstad (1984):

$$
\mathrm{ARM}=0,0112 \mathrm{RR}+0,00031 \mathrm{RR}^{2}-0,0012 \mathrm{RR} \mathrm{S}_{0}
$$

em que RR - rugosidade randômica, m; e $\mathrm{S}_{0}$ - declividade da superfície do solo, \%.

O termo máximo é usado porque existem casos em que o armazenamento superficial não será completamente preenchido. 
A rugosidade randômica é estimada pela equação proposta por Alberts et al. (1995):

$$
\mathrm{RR}_{\mathrm{i}}=\mathrm{RR}_{0} \mathrm{~T}_{\mathrm{ds}}+\mathrm{RR}_{\mathrm{t}-1}\left(1-\mathrm{T}_{\mathrm{ds}}\right)
$$

em que $\mathrm{RR}_{\mathrm{i}}$ - rugosidade randômica imediatamente após o preparo do solo, $\mathrm{m} ; \mathrm{RR}_{0}$ - rugosidade randômica criada pelo implemento de preparo, $\mathrm{m} ; \mathrm{T}_{\mathrm{ds}}$ - fração da superfície do solo mobilizada pelo implemento de preparo, \%; e $\mathrm{RR}_{\mathrm{t}-1}$ - rugosidade randômica do solo no dia anterior à operação de preparo, $\mathrm{m}$.

Os valores de $\mathrm{RR}_{0}$ e $\mathrm{T}_{\mathrm{ds}}$ usados no modelo são aqueles recomendados por Alberts et al. (1995). A rugosidade randômica da superfície do solo no dia anterior à operação de preparo do solo é obtida de um banco de dados visual apresentado por Renard et al. (1997).

O tempo para o qual toda a capacidade de armazenamento de água sobre a superfície do solo é preenchida $t_{\mathrm{PRE}}$ é calculado pela equação:

$$
\mathrm{ARM}=\int_{\mathrm{t}_{\mathrm{i}(\mathrm{ARM})}}^{\mathrm{t}_{\mathrm{PRE}}}\left(\mathrm{i}_{\mathrm{i}}-\mathrm{VI}\right) \mathrm{dt}
$$

em que $t_{\mathrm{PRE}}$ - tempo para o qual a capacidade de armazenamento de água sobre a superfície do solo se encontra preenchida, $\min$.

O escoamento superficial começa quando a capacidade de armazenamento superficial é preenchida. A taxa de escoamento superficial, é expressa pela equação:

$$
\mathrm{q}_{\mathrm{ES}}=\mathrm{i}_{\mathrm{i}}-\mathrm{VI}
$$

em que $\mathrm{q}_{\mathrm{ES}}$ - taxa de ocorrência do escoamento superficial, $\mathrm{mm} \mathrm{h}^{-1}$.

A velocidade de infiltração durante o intervalo de tempo em que o escoamento superficial ocorre é calculada pela Eq. 8 até o momento $\left(\mathrm{t}_{\mathrm{f}(\mathrm{ES})}\right)$ em que $\mathrm{i}_{\mathrm{i}}=\mathrm{VI}$. Este tempo é obtido pela equação:

$$
\frac{\mathrm{K} \mathrm{T}^{\mathrm{a}}}{\left(\mathrm{t}_{\mathrm{f}(\mathrm{ES})}+\mathrm{b}\right)^{\mathrm{c}}}\left(1-\frac{\mathrm{ct}_{\mathrm{f}(\mathrm{ES})}}{\mathrm{t}_{\mathrm{f}(\mathrm{ES})}+\mathrm{b}}\right)=\mathrm{K}_{\mathrm{e}}\left(1+\frac{\left(\theta_{\mathrm{s}}-\theta_{\mathrm{i}}\right) \mathrm{S}_{\mathrm{w}}}{\mathrm{I}}\right)
$$

em que $t_{f(E S)}$ - tempo no qual o escoamento superficial termina, $\min$.

Após $\mathrm{t}_{\mathrm{f}(\mathrm{ES})}$, mesmo se $\mathrm{i}_{\mathrm{i}}$ é menor que VI, VI continua a ser obtida pela Eq. 8. Esta condição é mantida até que toda a lâmina de armazenamento superficial se infiltre no solo, isto é, até que:

$$
\int_{t_{f(E S)}}^{t_{f(A R M)}}\left(V I-i_{i}\right) d t=A R M
$$

em que $\left(\mathrm{t}_{\mathrm{f}(\mathrm{ARM})}\right)$ - tempo no qual toda a lâmina de armazenamento superficial se infiltra no solo, min.

A partir deste momento, a velocidade de infiltração torna-se igual a $i_{i}$ e a infiltração acumulada é calculada pela Eq. 7 .

A precipitação total (PT) que ocorre durante o evento considerado, com duração $\mathrm{t}$, em mm, é obtida pela equação:

$$
P T=\int_{0}^{t} i_{i} d t
$$

A infiltração acumulada (I), em mm, é obtida pela soma da infiltração que ocorre durante as diferentes fases associadas ao balanço hídrico sendo, desta forma, expressa pela equação

$$
I=\int_{t_{(I C V)}}^{t_{i}(\text { ARM })} i_{i} d t+\int_{t_{i(A R M)}}^{t_{f(A R M)}} V I d t+\int_{t_{f(A R M)}}^{t} i_{i} d t
$$

Se a precipitação termina antes que a lâmina total de armazenamento superficial se infiltre, a infiltração continuará a ser expressa pela Eq. 8, até que todo o armazenamento superficial se infiltre no solo. Neste caso, é necessário considerar-se esta lâmina infiltrada (ocorrida após o fim da precipitação) na Eq. 16.

A lâmina de escoamento superficial (ES) em mm é calculada pela equação:

$$
\mathrm{ES}=\mathrm{PT}-\mathrm{ICV}-\mathrm{I}
$$

O armazenamento superficial não é considerado na Eq. 17, porque ele é transformado em infiltração.

Antes que ocorra a percolação profunda, a lâmina infiltrada deve elevar a umidade do solo até a capacidade de campo. A quantidade de água requerida para elevar a umidade do solo à capacidade de campo é calculada pelas equações:

$$
\begin{aligned}
& \mathrm{DEF}=\mathrm{L}_{\mathrm{CC}}-\mathrm{L}_{\mathrm{SWA}} \\
& \mathrm{L}_{\mathrm{CC}}=\left(\theta_{\mathrm{CC}} \mathrm{D}_{\mathrm{Z}}\right) 10 \\
& \mathrm{~L}_{\mathrm{SWA}}=\left(\theta_{\mathrm{a}} \mathrm{D}_{\mathrm{Z}}\right) 10
\end{aligned}
$$

em que DEF - déficit de água no solo em relação à capacidade de campo, $\mathrm{mm} ; \mathrm{L}_{\mathrm{cc}}$ - quantidade de água presente no solo quando este se encontra na capacidade de campo, mm; $\mathrm{L}_{\mathrm{SWA}}$ - quantidade de água presente no solo quando este se encontra na sua umidade atual, $\mathrm{mm} ; \theta_{\mathrm{CC}}$ - capacidade de campo, em base de volume, $\mathrm{cm}^{3}$ de água $\mathrm{cm}^{-3}$ de solo; $\theta_{\text {a }}$ - umidade atual do solo, em base de volume, $\mathrm{cm}^{3}$ de água $\mathrm{cm}^{-3}$ de solo; e $\mathrm{D}_{\mathrm{Z}}$ - profundidade do sistema radicular, $\mathrm{cm}$.

A percolação profunda, $\mathrm{P}_{\mathrm{P}}$, em mm, é determinada por:

$$
\mathrm{P}_{\mathrm{P}}=\max (0, \mathrm{I}-\mathrm{DEF})
$$


Com base nesses dados, a umidade de água presente no solo pode ser obtida pela equação:

$$
\mathrm{L}_{\mathrm{SWA}_{\mathrm{t}}}=\mathrm{L}_{\mathrm{SWA}_{\mathrm{t}-1}}+\mathrm{I}-\mathrm{ET}-\mathrm{P}_{\mathrm{P}}
$$

em que ET é a evapotranspiração, mm.

Se o projetista pretende obter o escoamento superficial para um período de retorno específico, este deve ser inserido na Eq. 6; entretanto, se o cálculo é para uma condição predefinida (condição na qual o total precipitado e a duração da precipitação são conhecidos) será necessário, então, obterse o período de retorno correspondente a esta situação pela Eq. 6 e, então, aplicar o procedimento apresentado. Esta situação apresenta um grande potencial de aplicação em condições em que já se tem uma previsão do total precipitado e da duração associada a esta precipitação. Com esses dados, estima-se o período de retorno com o emprego da Eq. 6 e pode-se estimar cada um dos componentes associados ao balanço hídrico para este evento.

\section{APLICAÇÕES PRÁTICAS E DISCUSSÃO}

Considerando-se que o escoamento superficial integra todos os processos simulados pelo modelo, o esforço inicial para validar o modelo baseou-se somente em dados obtidos para o escoamento superficial. Dados coletados por Martins Júnior (1997) em 12 testes desenvolvidos em condições de chuva simulada em um solo sem cobertura vegetal e com declividade de $9,5 \%$, foram usados. Nestas condições, a interceptação pela cobertura vegetal é nula. A umidade do solo no momento da aplicação da chuva era muito próxima à umidade de saturação (condição em que a velocidade de infiltração é muito próxima à velocidade de infiltração básica da água no solo). Os valores de escoamento superficial obtidos experimentalmente e simulados são apresentados na Tabela 1, enquanto os valores de lâmina de escoamento superficial obtidos usando-se o método do balanço hídrico proposto neste trabalho apresentaram variações menores que $12 \%$ em relação aos valores medidos, e o coeficiente de correlação entre os valores de escoamento superficial simulados e medidos foi 0,985 .
Duas aplicações da metodologia desenvolvida são apresentadas na seqüência.

\section{Exemplo 1}

1. Equação de intensidade, duração e freqüência da precipitação para Uberaba, Minas Gerais, Brasil

$$
i_{m}=\frac{3000 T^{0,206}}{(t+37,459)^{0,904}}
$$

2. Características do solo: $\mathrm{K}_{\mathrm{s}}=20 \mathrm{~mm} \mathrm{~h}^{-1} ; \theta_{\mathrm{s}}=0,35 \mathrm{~cm}^{3} \mathrm{~cm}^{-3}$; $\theta_{\mathrm{i}}=0,20 \mathrm{~cm}^{3} \mathrm{~cm}^{-3} ; \theta_{\mathrm{CC}}=0,23 \mathrm{~cm}^{3} \mathrm{~cm}^{-3} ; \mathrm{S}_{\mathrm{w}}=70 \mathrm{~mm} \mathrm{e} \mathrm{D}_{\mathrm{Z}}=60 \mathrm{~cm}$

3. Evapotranspiração $(E T)=4,5 \mathrm{~mm} \mathrm{~d}^{-1}$ (calculada pelo modelo de Ritchie)

4. Período de retorno $(\mathrm{T})=10$ anos

5. Duração da chuva $(\mathrm{t})=60 \mathrm{~min}$

6. Vegetação: grama

7. Interceptação máxima pela cobertura vegetal, obtida no banco de dados apresentado por Woolhiser et al. (1990) e Morgan et al. (1998) para a grama $=2,0 \mathrm{~mm}$

8. Declividade do solo $=2 \%$

A Figura 1 mostra que, até um tempo igual a 0,7 min, calculado pela Eq. 4, toda a precipitação é interceptada pela vegetação e, após este tempo, a chuva é completamente transformada em infiltração, sendo $\mathrm{VI}=\mathrm{i}_{\mathrm{i}}$. Esta condição é mantida até que $i_{i}($ Eq. 5) supere a velocidade de infiltração (Eq. 8).

O empoçamento sobre a superfície do solo começa no tempo $t_{\mathrm{i}(\mathrm{ARM})}=1,1$ min e a velocidade de infiltração começa a ser expressa pela equação de Green-Ampt modificada por Mein-Larson (Eq. 8); desta forma, o armazenamento superficial inicia somente no momento em que a intensidade instantânea de precipitação (Eq. 5) supera a velocidade de infiltração (Eq. 8).

O máximo armazenamento superficial, calculado usando-se a metodologia proposta por Onstad (1984), foi igual a 12,6 min e o tempo para preencher o armazenamento superficial $\left(\mathrm{t}_{\mathrm{PRE}}\right)$ calculado pela Eq. 11, foi igual a 8,9 min. O escoamento superficial começa neste tempo e sua taxa de ocorrência é expressa pela Eq. 12. Mesmo após a chuva ter parado, com

\begin{tabular}{|c|c|c|c|c|c|c|}
\hline \multirow{2}{*}{ Teste } & \multirow{2}{*}{$\begin{array}{l}\text { Intensidade de } \\
\text { Precipitação } \\
\left(\mathrm{mm} \mathrm{h}^{-1}\right)\end{array}$} & \multirow{2}{*}{$\begin{array}{c}\text { Duração do } \\
\text { Experimento } \\
\text { (min) }\end{array}$} & \multirow{2}{*}{$\begin{array}{c}\mathrm{VIB}^{*} \\
\left(\mathrm{~mm} \mathrm{~h}^{-1}\right)\end{array}$} & ESmedido & ESsimulado & \multirow{2}{*}{$\begin{array}{c}\text { ESsimulado/ESmedido } \\
(\%)\end{array}$} \\
\hline & & & & \multicolumn{2}{|c|}{$(\mathrm{mm})$} & \\
\hline 1 & 94,2 & 24 & 28,9 & 29,1 & 26,5 & 91,1 \\
\hline 2 & 74,6 & 40 & 23,4 & 36,8 & 35,6 & 96,7 \\
\hline 3 & 94,3 & 24 & 29,3 & 27,9 & 26,3 & 94,3 \\
\hline 4 & 119,0 & 14 & 29,4 & 19,8 & 19,2 & 97,0 \\
\hline 5 & 96,9 & 24 & 21,2 & 31,1 & 29,6 & 95,2 \\
\hline 6 & 76,9 & 40 & 28,0 & 31,8 & 32,6 & 102,5 \\
\hline 7 & 118,9 & 14 & 21,7 & 19,8 & 21,0 & 106,1 \\
\hline 8 & 117,7 & 14 & 29,3 & 19,0 & 19,3 & 101,6 \\
\hline 9 & 94,8 & 24 & 19,2 & 29,5 & 30,4 & 103,1 \\
\hline 10 & 75,6 & 40 & 18,5 & 35,9 & 38,9 & 108,4 \\
\hline 11 & 74,3 & 40 & 16,1 & 36,4 & 40,5 & 111,3 \\
\hline 12 & 119,8 & 14 & 31,3 & 18,9 & 18,8 & 99,5 \\
\hline
\end{tabular}

Tabela 1. Comparação entre as lâminas totais de escoamento superficial (ES), simulada e medida 
(A)

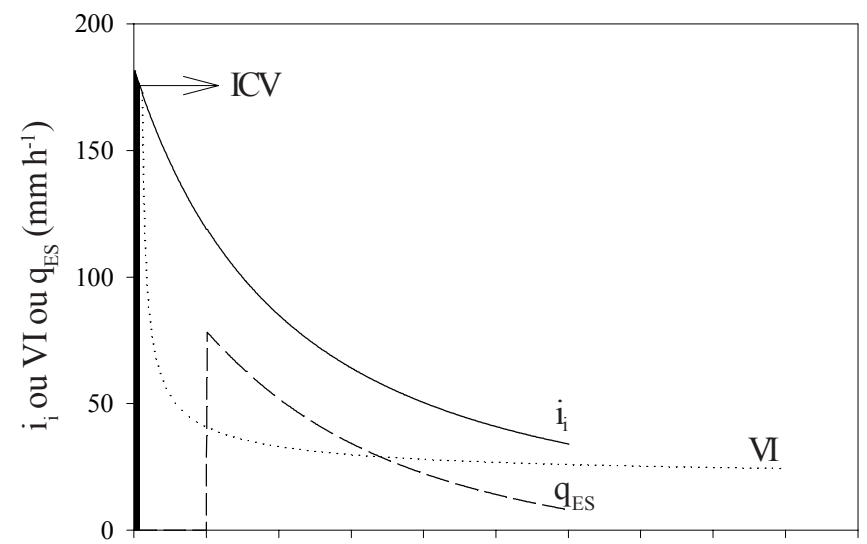

(B)

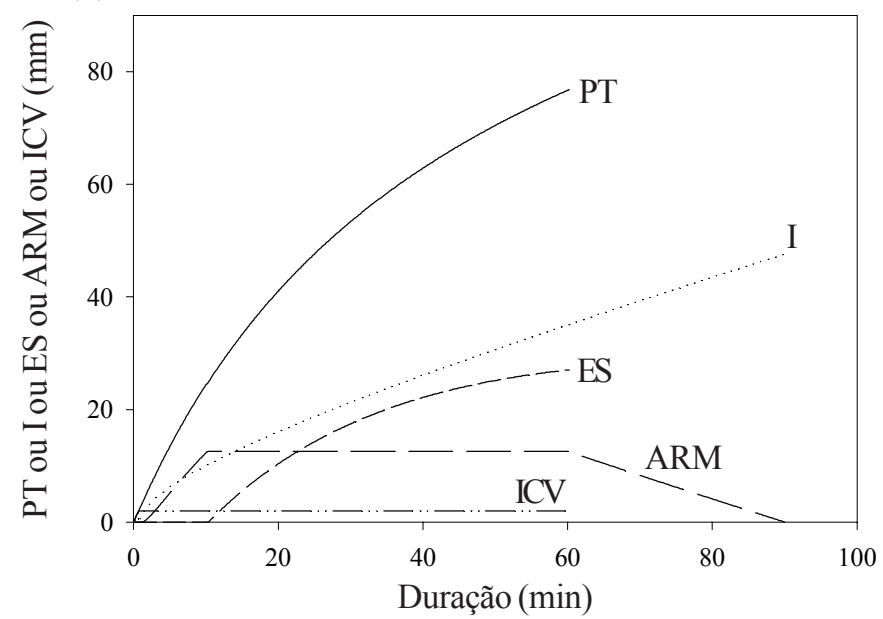

Figura 1. Representação dos componentes associados à aplicação do modelo proposto para o Exemplo 1: (A) expressos em termos de taxa e (B) expressos em termos de lâmina

60 min a infiltração continua até $90 \mathrm{~min}$. Durante este tempo adicional, a fração do armazenamento superficial que estava presente na superfície do solo quando a precipitação terminou infiltra. A velocidade de infiltração durante o período de tempo em que o escoamento superficial ocorre continua a ser expressa pela Eq. 8 até o momento $\left(\mathrm{t}_{\mathrm{f}(\mathrm{ES})}\right)$ em que $\mathrm{i}_{\mathrm{i}}=$ VI. Este tempo é obtido pela Eq. 13. Neste exemplo, o escoamento superficial termina somente quando a precipitação também termina.

O total precipitado durante o evento considerado, com duração t, é obtido pela Eq. 15 e é igual a 76,8 mm. A infiltração acumulada, $47,7 \mathrm{~mm}$, é obtida pela soma da lâmina infiltrada durante as diversas fases associadas ao balanço hídrico, e é expressa pela Eq. 16, enquanto o volume escoado superficialmente, $27,1 \mathrm{~mm}$, é calculado pela Eq. 17. A lâmina de percolação profunda, calculada pela Eq. 21, é de $29,7 \mathrm{~mm}$, e a lâmina de água presente no solo na condição estudada (Eq. 22) é de $133,5 \mathrm{~mm}$.

\section{Exemplo 2}

Neste segundo exemplo, as condições definidas são as mesmas do exemplo 1, mas o tempo considerado é de $180 \mathrm{~min}$, o período de retorno é de 2 anos e a evapotranspiração é de $3,5 \mathrm{~mm} \mathrm{~d}^{-1}$ (calculada pelo modelo de Ritchie). Neste caso,
Figura 2, a máxima interceptação pela cobertura vegetal é a mesma que a do exemplo $1(2,0 \mathrm{~mm})$ mas o tempo necessário para preencher a máxima interceptação pela cobertura vegetal é igual a $0,9 \mathrm{~min}$ (no primeiro exemplo este valor foi $0,7 \mathrm{~min}$ ). O tempo necessário para preencher a máxima interceptação pela cobertura vegetal é maior porque a intensidade da precipitação é mais baixa (período de retorno mais baixo que a do exemplo 1).

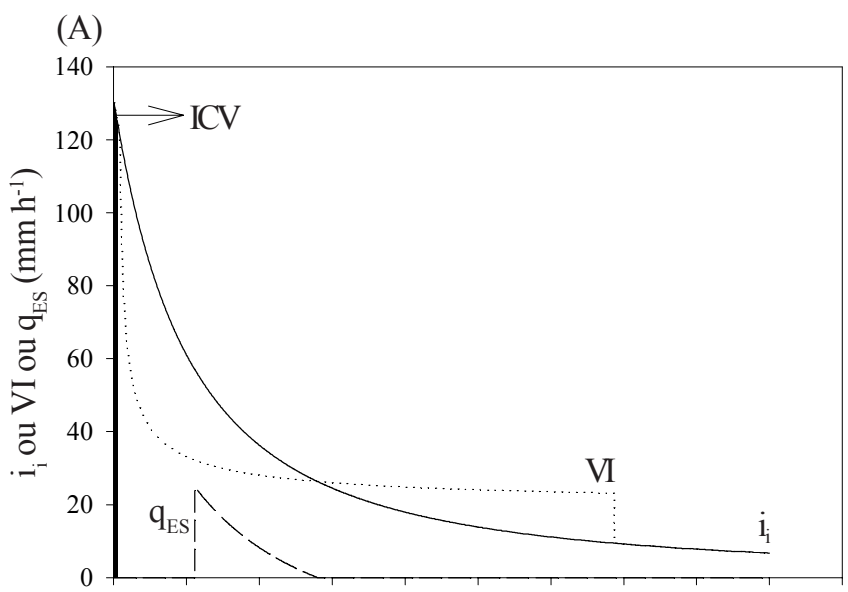

(B)

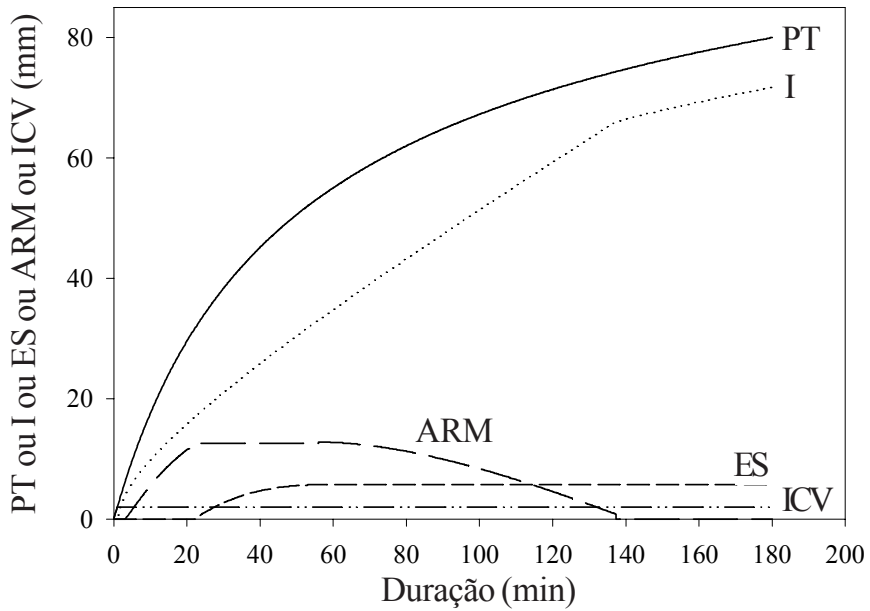

Figura 2. Representação dos componentes associados à aplicação do modelo proposto para o Exemplo 2: (A) expressos em termos de taxa e (B) expressos em termos de lâmina

O empoçamento de água sobre a superfície do solo começa no tempo $t_{\mathrm{i}(\mathrm{ARM})}=1,0$ min e a velocidade de infiltração começa a ser expressa pela equação GAML (Eq. 8). O máximo armazenamento superficial é $12,6 \mathrm{~mm}$, mesmo valor que no exemplo 1, mas o tempo para o qual toda a capacidade de armazenamento superficial é preenchida $\left(\mathrm{t}_{\mathrm{PRE}}\right)$ (calculada pela Eq. 11) é 21,1 min (no exemplo 1 este valor foi $8,9 \mathrm{~min}$ ). $\mathrm{O}$ escoamento superficial tem início neste tempo, e é expresso pela Eq. 12.

A velocidade de infiltração durante o período em que ocorre escoamento superficial continua a ser expressa pela Eq. 8 até o momento $\left(\mathrm{t}_{\mathrm{f}(\mathrm{ES})}\right)$ em que $\mathrm{i}_{\mathrm{i}}=\mathrm{VI}$. Este tempo é obtido pela Eq. 13 e, no caso, é igual a 55,8 min. A partir do momento em que o escoamento superficial termina, a velocidade de infiltração continua sendo expressa pela Eq. 8, mesmo quando $\mathrm{i}_{\mathrm{i}}$ é menor que VI, condição esta mantida até que ocorra a infiltração de 
toda a lâmina armazenada na superfície do solo $\left(\mathrm{t}_{\mathrm{f}(\mathrm{ARM})}\right) \mathrm{e}, \mathrm{a}$ partir deste momento, a velocidade de infiltração torna-se igual à intensidade instantânea de precipitação. No exemplo 2, o valor de $\mathrm{t}_{\mathrm{f(ARM})}$ é igual a 137,3 min.

O total precipitado durante o evento considerado, com duração t, é obtido pela Eq. 15 e é igual a $80 \mathrm{~mm}$. A infiltração acumulada, 72,6 mm, é obtida pela soma da lâmina infiltrada durante as diversas fases associadas ao balanço hídrico, e é expressa pela Eq. 16, enquanto a lâmina de escoamento superficial, 5,5 mm, é calculada pela Eq. 17. A lâmina de percolação profunda (Eq. 21) é de 54,6 mm e a lâmina de água armazenada no solo no dia considerado (Eq. 22) é de 134,5 mm.

\section{CONCLUSÕES}

1. A disponibilidade de informações da equação de intensidade, duração e freqüência da precipitação para diversos estados brasileiros, e o fato do modelo apresentar um evidente embasamento físico, potencializam a utilização desta metodologia em substituição a outros modelos empíricos desenvolvidos em países com condições muito diferenciadas daquelas existentes no Brasil.

2. O modelo pode ser utilizado tanto para a estimativa de valores máximos (sobretudo do escoamento superficial), de grande necessidade no dimensionamento de estruturas hidráulicas, como para o cálculo contínuo do balanço hídrico, a fim de monitoramento ambiental ou, até mesmo, para o manejo de sistemas de irrigação.

3. Os resultados obtidos da simulação com o modelo foram muito próximos daqueles observados no campo.

\section{LITERATURA CITADA}

Alberts, E.E.; Nearing, M.A.; Weltz, L.; Risse, M.; Pierson, F.B.; Soil component. In: Flanagan, D.C.; Nearing, M.A. (ed.) Water Erosion Prediction Project (WEPP) - Technical Documentation. NSERL Report n. 10. West Lafayette: USDA/NSEAL, 1995. cap. 3. 18p.

Baker, C.H.; Horrockx, R.D.; Goering, C.E. Use of the Gompertz function for predicting corn leaf area. Transaction of the ASAE, St. Joseph, v.18, n.2, p.323-326, 1975.

Bonta, J.V.; Rao, A.R. Estimating peak flows from small agricultural watersheds. Journal of Irrigation and Drainage Engineering, New York, v.118, n.1, p.122-137, 1992.

$\mathrm{Chu}, \mathrm{S}$.T. Infiltration during an unsteady rain. Water Resources Research, Washington, v.14, n.3, p.461-466. 1978.

Ehlers, W. Transpiration efficiency of oat. Agronomy Journal, Madison, v.81, p.810-817. 1989.

Flanagan, D.C.; Nearing, M.A. (ed.) Water Erosion Prediction Project (WEPP) - Technical Documentation. NSERL Report n. 10. West Lafayette: USDA/NSEAL, 1995. n.p.

Knisel, W.G. (Ed.). CREAMS: A field-scale model for chemicals, runoff, and erosion from agricultural management systems. U.S. Department of Agriculture, Science and Education Administration, Conservation Research Report N.26. 643p. 1980.

Linsley Jr., R.K.; Kohler, M.A.; Paulhus, J.L.H. Hydrology for engineers. New York: McGraw-Hill Book Co., Inc., 1975.

Martins Junior, D. Avaliação de um modelo de simulação da lâmina e vazão máximas de escoamento superficial. Viçosa: UFV 1997. 86p. Dissertação Mestrado
Mein, R.G.; Larson, C.L. Modeling infiltration during a steady rain. American Geophysical Union, Washington, v.9, n.2, p.384-394. 1973.

Mohamoud, Y.M.; Ewing, L.K.; Boast, C.W. Small plot hydrology: I. Rainfall infiltration and depression storage determination. Transactions of the ASAE, St. Joseph, v.33, n.4,p.1121-1131. 1990.

Morgan, R.P.C.; Quinton, J.N.; Smith, R.E. EUROSEM: Documentation and user guide. Version 3.6. Silsoe. 1998.

Onstad, C.A. Depression storage on tillage soil surfaces. Transactions of the ASAE, St. Joseph, v.27, n.3, p.729-732. 1984.

Pathak, P.; Laryea, K.B.; Sudi, R. A runoff model for small watersheds in the semi-arid tropics. Transactions of the ASAE, St. Joseph, v.32, n.5, p.1619-1624. 1989.

Potter, K.N. Soil properties effect on random roughness decay by rainfall. Transactions of the ASAE, St. Joseph, v.33, n.6, p.1899-1892. 1990.

Pruski, F.F.; Calijuri, M.L.; Bhering, E.M.; Silva, J.M.A. Metodologia baseada no uso de sistemas de informações geográficas para a obtenção de equações de chuvas intensas em qualquer localidade do Estado do Paraná. Engenharia na Agricultura, Viçosa, v.5, n.3, p.254-265. 1997a.

Pruski, F.F.; Ferreira, P.A.; Ramos, M.M.; Cecon., P.R. Model to design level terraces. Journal of Irrigation and Drainage Engineering, New York, v.23, n.1, p.8-12. 1997 b.

Pruski, F.F.; Silva, D.D.; Braga, J.C.; Rodrigues, L.N.; Silva, J.M.A.; Griebeler, N.P.; Teixeira, A.F.; Santos, W.L.; Baena, L.G.N.; Chaves, M.A.O. HIDROS: Sistema para o planejamento e manejo integrado dos recursos hídricos - manual do usuário. Brasília, MMA/SRH/UFV/FUNARBE, 1999. 111p.

Renard, K.G.; Foster, G.R.; Weesies, G.A.; McCool, D.K.; Yoder, D.C. Predicting soil erosion by water: a guide to conservation planning with the revised universal soil loss equation (RUSLE) - Agricultural handbook, 703. Tucson: USDA/ARS. 1997.

Schwab, G.O.; Frevert, R.K.; Edminster, T.W.; Barnes, K.K. Soil and water conservation engineering, 3 ed., New-York: John Wiley \& Sons, Inc., 1981. 683p.

Silva, D.D.; Pinto, F.R.L.P.; Pruski, F.F.; Pinto, F.A. Estimativa e espacialização dos parâmetros da equação de intensidadeduração-freqüência da precipitação para os Estados do Rio de Janeiro e Espírito Santo. Engenharia Agrícola, Jaboticabal, v.18, n.3, p.11-21, 1999a.

Silva, D.D.; Valverde, A.E.L.; Pruski, F.F.; Gonçalves, R.A.B. Estimativa e espacialização dos parâmetros da equação de intensidade-duração-freqüência da precipitação para o Estado de São Paulo. Engenharia na Agricultura, Viçosa, MG, v.7, n.2, p.70-87, 1999b.

Slack, D.C. Modeling infiltration under moving sprinkler irrigation systems. Transactions of the ASAE, St. Joseph, v.23, n.3, p.596-600. 1980.

Stone, J.; Renard, K.G.; Lane, L.J. Runoff estimation on agricultural fields. In: Agassi, M. (ed.) Soil erosion, conservation, and rehabilitation. New York: Marcel Dekker, 1996. Cap. 10, p.203-238.

Woolhiser, D.A.; Smith, R.E.; Goodrich, D.C. KINEROS: A kinematic runoff and erosion model: documentation and user manual. USDA - Agricultural Research Service, ARS-77. 1990. 130p. 